Published in final edited form as:

Clin Lung Cancer. 2017 May ; 18(3): e151-e159. doi:10.1016/j.cllc.2017.01.010.

\title{
A Multimedia Self-Management Intervention to Prepare Cancer Patients and Family Caregivers for Lung Surgery and Post- Operative Recovery
}

\author{
Virginia Sun, PhD, RN ${ }^{a}$, Dan J. Raz, MD ${ }^{b}$, Nora Ruel, MA ${ }^{c}$, Walter Chang, ${ }^{d}{ }^{d}$, Loretta \\ Erhunmwunsee, MD $^{\mathrm{b}}$, Karen Reckamp, MD $^{\mathrm{e}}$, Brian Tiep, MD $^{f}$, Betty Ferrell, PhD, FAAN ${ }^{\mathrm{a}}$, \\ Ruth McCorkle, PhD, RN, FAAN ${ }^{\text {, }}$, and Jae Y. Kim, MD ${ }^{\mathrm{b}}$ \\ aDivision of Nursing Research and Education, Department of Population Sciences, City of Hope, \\ 1500 East Duarte Road, Duarte, CA \\ ${ }^{b}$ Division of Thoracic Surgery, Department of Surgery, City of Hope, 1500 East Duarte Road, \\ Duarte, CA \\ 'Biostatistics Core, Department of Information Sciences, City of Hope, 1500 East Duarte Road, \\ Duarte, CA \\ ${ }^{\mathrm{d} D e p a r t m e n t}$ of Anesthesiology, City of Hope, 1500 East Duarte Road, Duarte, CA \\ eDepartment of Medical Oncology and Therapeutics Research, City of Hope, 1500 East Duarte \\ Road, Duarte, CA \\ fDepartment of Respiratory Diseases and Pulmonary Rehabilitation, City of Hope, 1500 East \\ Duarte Road, Duarte, CA \\ gSchool of Nursing, Yale University, 400 West Campus Drive, West Haven, CT
}

\begin{abstract}
Purpose-To evaluate the feasibility and acceptability of a multimedia self-management (MSM) intervention to prepare patients and family caregivers for lung surgery.
\end{abstract}

Patients and Methods-This is a quasi-experimental, two-group, sequential enrollment pilot study of a four-session multimedia intervention (audio/visual + print) to enhance self-management and QOL for patients and family caregivers. The intervention, Preparing for Lung Surgery, begins before surgery, and continues through hospitalization and discharge, with two telephone support sessions after discharge. Outcomes were assessed before surgery (pre-intervention), at discharge, and 2-4 weeks post-discharge (post-intervention). Patient outcomes were assessed using the FACT-G (QOL), MDASI and FACT-PSI (symptoms), self-efficacy, surgery-related knowledge,

\footnotetext{
Correspondence: Virginia Sun, PhD, RN, Assistant Professor, Division of Nursing Research and Education, Department of Population Sciences, City of Hope, 1500 East Duarte Road, Duarte, CA 91010, USA; Tel: 626/256-4673 ext. 63122; fax: 626/301-8941; vsun@coh.org.

Dr. Sun and Dr. Kim contributed equally to the conception and design of the study.

Publisher's Disclaimer: This is a PDF file of an unedited manuscript that has been accepted for publication. As a service to our customers we are providing this early version of the manuscript. The manuscript will undergo copyediting, typesetting, and review of the resulting proof before it is published in its final citable form. Please note that during the production process errors may be discovered which could affect the content, and all legal disclaimers that apply to the journal pertain.
} 
and patient activation. Family caregiver outcomes included COH-QOL-Family (QOL), Caregiver Burden Scale, and knowledge. Paired $t$-tests were used for exploratory evaluations of score changes from pre- to post-intervention.

Results-Sixty participants (38 patients, 22 family caregivers) enrolled in the study (70\% accrual). Post-intervention scores were significantly improved for patients' emotional QOL $(\mathrm{p}=0.001)$. Trends for improvements were observed for patient self-efficacy, surgery-related knowledge, activation. Family caregivers' surgery-related knowledge was significantly improved $(\mathrm{p}=0.02)$. Overall, participants were highly satisfied with the acceptability/usability of the intervention (3.6 to 3.7/4.0).

Conclusion-A standardized multimedia self-management intervention was feasible and acceptable in supporting readiness and preparedness for lung surgery and postoperative recovery. A larger randomized trial is needed to verify the impact of the MSM intervention on patient/family caregiver outcomes and healthcare resource use.

\section{MicroAbstract}

This study pilot-tested a multimedia self-management (MSM) intervention for lung surgery patients and family caregivers. We administered the intervention to 60 patients/family caregivers from before surgery to 2-4 weeks after. Trends for improvements were observed in scores for selfefficacy, knowledge, activation, and emotional QOL. Patient-centered models of surgical care are needed to improve QOL and reduce undesirable healthcare resource use.

\section{Keywords}

Quality of life; lung cancer; self-management; family caregivers; multimedia; surgery

\section{Introduction}

Surgery is an essential component of curative therapy for lung cancer. The majority of patients with early stage lung cancer $(70 \%)$ undergo surgery. ${ }^{1}$ Surgical procedures are complex and invasive; patients often suffer from an abrupt increase in unpleasant symptoms, impaired functional status, and poor quality of life (QOL) after surgery. ${ }^{2}{ }^{3}$ Historically, the use of increasingly radical oncologic surgery to improve cure rates was the primary goal for cancer surgeons. ${ }^{4}$ Recent technological advances resulted in the rapid uptake of minimally invasive procedures to treat lung malignancies. Due to these advances in surgical care, as well as changes in the healthcare environment, patients are discharged from the hospital earlier after lung surgery. This is despite an aging population that suffers from greater comorbidities (pulmonary, cardiovascular) and impaired physical functioning. Consequently, the majority of postoperative recovery occurs in the community, with family caregivers providing the majority of care at home.

Surgical patients and family caregivers experience multiple physical and psychological stress prior to resection. ${ }^{5}$ This begins with the treatment decision-making process, where surgeons provide an explanation of the proposed procedure, risks, benefits, and postoperative recovery. Consequently, patients and families can be easily overwhelmed and experience challenges in retaining the information provided. Studies have shown that 
preoperative education could help patients and families cope with the stressful events, alleviate anxiety, increase self-efficacy, and support postoperative recovery activities. ${ }^{6}$ However, most of the evidence focused on general surgery. 6,7

Family caregivers frequently report unmet needs in all QOL domains. Our previous research suggests that lung cancer family caregivers experience significant psychological distress, higher caregiver burden, and decreased QOL related to their caregiving role. ${ }^{8-11}$ Patients and families often experience heightened feelings of powerlessness before and after surgery. ${ }^{12,} 13$ The rapid transition from postoperative hospitalization to self-management at home is often threatened by unmet discharge needs. ${ }^{14}$ Patients and families are often expected to take responsibility for managing recovery at home while still experiencing the physical and psychological effects of surgery. ${ }^{15,16}$ As postoperative care continues to move further into the home setting, lung surgery patients and family caregivers may be ill-prepared for recovery at home. This can potentially result in significant morbidity, undesirable healthcare resource use, and immense social and economic costs. The current literature offers few patient- and family-centered models of care in lung surgery. The purpose of this pilot study was to evaluate the feasibility and acceptability of a multimedia self-management (MSM) intervention to prepare patients and family caregivers for lung surgery.

\section{Patients and Methods}

\section{Study and Intervention Design}

This was a quasi-experimental, two-group, sequential enrollment pilot study of the MSM intervention, Preparing for Lung Surgery. The systematic development and content of the intervention was described elsewhere. ${ }^{17}$ Briefly, the MSM intervention is a multicomponent, multimedia model of care designed specifically for lung surgery. It provides one-on-one coaching with cancer patients and family caregivers, targeting knowledge and skills in symptom management, self-care, and post-operative recovery activities. The intervention includes video, handbook, and phone calls at home after hospital discharge. The inclusion of different media provides participants with alternative modes of learning. A recent meta-analysis found that video interventions in cancer were as effective, and in some RCTs, superior in knowledge transfer to print materials alone. ${ }^{18}$ Content in the video and handbook are identical, with more details provided within the print materials. The handbook was designed to complement the video to enhance pre-operative preparation and postoperative recovery activities.

The intervention is based on the Chronic Care Self-Management Model (CCM). ${ }^{19-21}$ The CCM improves patient and family caregiver outcomes through proactive planning, knowledge enhancement, self-efficacy (confidence in self-management) and activation (confidence in managing and coordinating overall healthcare). ${ }^{22-24}$ It is also defined within the context of the family, and recognizes the unique self-management needs of FCGs. ${ }^{16,25}$ The MSM intervention combines both traditional (information and technical skills) and selfmanagement education (enhance activation and self-efficacy). ${ }^{17}$ Intervention content is divided into four sessions (Table 1). Session 1 focuses on what to expect before surgery and during hospitalization after surgery. Session 2 focuses on what to expect after discharge. Sessions 3 and 4 involves two telephone supports after discharge. 


\section{Sample and Setting}

Patients with confirmed diagnoses of lung cancer or a secondary malignancy of the lung, who were English-speaking and scheduled to undergo surgery, were eligible for participation in the study. Once enrolled, patients were asked to identify a family caregiver to participate in the study. Family caregivers were defined as either a family member or friend who provided the majority of care after surgery. Patients who did not have an identified family caregiver were included in the study. The rationale for inclusion of both patients with and without a family caregiver was for practical purposes to complete the pilot study within a 12-month timeframe. Participants were recruited from the thoracic surgery outpatient clinic of a National Cancer Institute-designated comprehensive cancer center located in Southern California.

\section{Participant Outcome Measures}

Patient QOL was assessed using the Functional Assessment of Cancer Therapy tool, which contains 27-items that measures physical, social/family, emotional, and functional wellbeing. All items are scored on a 5-point Likert scale ( $0=$ not at all; $4=$ very much). ${ }^{26}$ The MD Anderson Symptom Inventory (MDASI) and FACT-Pulmonary Symptom Index (PSI) were used to assess symptoms. The MDASI contains 13 symptom items and assesses the symptom severity and impact on daily functioning (0-10 scale) ${ }^{27}$ The PSI contains 4 items that assessed dyspnea, cough, chest tightness, and ease with breathing on a 5-point Likert scale. ${ }^{28}$ Self-efficacy was assessed using a modified, 8-item version of the Self-Efficacy Scale developed by Lorig and colleagues. ${ }^{29}$ The scale evaluates the patient's perceived confidence in performing self-care activities before and after surgery. Patient knowledge was assessed using a 10 item tool developed by the investigators to evaluate knowledge transfer on self-management before and after surgery. The Patient Activation Measure (PAM) assessed "activation", which reflects a patient's level of engagement and empowerment in their healthcare. ${ }^{23}$ Clinical, surgical, and healthcare resource use outcomes were obtained through a review of electronic medical chart records.

Family caregiver QOL was assessed with the City of Hope-Quality of Life-Family (COHQOL-Family). This 37-item tool measures QOL in the physical, psychological, social, and spiritual well-being domains. ${ }^{30,31}$ Caregiver burden was assessed with the Montgomery Borgatta Caregiver Burden Scale (MBCBS). This 14-item tool measures the impact of caregiving on three dimensions of burden: objective, subjective demand, and subjective stress. ${ }^{32}$ Family caregiver knowledge was assessed using a 10 item tool developed by the investigators to evaluate caregiver knowledge transfer on self-management before and after surgery. Finally, participants in the intervention group completed a satisfaction tool to assess usability and acceptability of the MSMI.

\section{Study Procedures}

Study procedures and protocol were approved by the Institutional Review Board. Eligible patients were identified and referred by the thoracic surgical team. Patients and family caregivers were invited to participate following decisions to undergo surgery for treatment or 3-7 days before surgery. All participants provided voluntary informed consent prior to enrollment. Participants were sequentially-enrolled, with patients/family caregivers in the 
control group enrolled first, followed by the intervention group. Upon enrollment, participants completed baseline surveys before surgery. Session 1 of the MSM intervention was delivered 3-7 days before surgery. In this session, the video was viewed by patients and family caregivers during a routine pre-operative outpatient visit. The intervention handbook was given to participants following viewing, and key contents were reviewed. After surgery, participants viewed the Session 2 video within 24 hours of planned discharge. Handbook content was reviewed following video viewing. Outcomes were assessed prior to discharge. Sessions 3 and 4 (telephone support) were delivered at day 2 and day 7 post-discharge. Outcomes were reassessed at the first post-operative outpatient visit (approximately 2-4 weeks post-discharge).

\section{Statistical Analysis}

Data from Cardiff teleforms were scanned into a relational database and audited for accuracy prior to analysis. Descriptive statistics were used to summarize participant demographics, clinical/surgical characteristics, outcomes, data abstracted from medical chart reviews, and acceptability/usability of the intervention, and Fisher's exact test or the Wilcoxon rank-sum test were used to compare discrete and continuous variables (respectively) between Control and Intervention groups. Established instruments were scored according to standard instructions, and appropriate descriptive statistics were computed. Cronbach's alpha was calculated for the PAM and Self-Efficacy Scales to assess reliability of those tools for our specific study population. We explored changes in outcome scores using paired $t$-tests from pre-intervention (baseline) to 2-4 weeks post-discharge (post-intervention).

\section{Results}

Of those eligible for the study in a 12-month period, an estimated $70 \%$ chose to participate in the study. The most common reasons for patients declining participation were being too overwhelmed (72\%) and severe illness (28\%). Being too busy (64\%) and too overwhelmed (36\%) were the most common reasons for family caregivers to decline participation. A total of 44 patients and 29 family caregivers $(\mathrm{N}=73)$ provided informed consent for study participation. Of this total, 6 patients and 7 family caregivers $(\mathrm{N}=13)$ dropped-out of the study (17.8\% attrition). Reasons for drop-out included severe illness (56\%), being overwhelmed (24\%), and no longer wanting to participate (20\%). After accounting for attrition, a total of 60 participants completed the study ( 38 patients, 22 family caregivers).

Mean age for patients was 66.8 for usual care and 64.5 for the intervention group. For family caregivers, mean age was 60.5 for usual care and 59.5 for intervention group. The majority of family caregivers (81.8\%) were female (Table 2). Demographic variables among patients varied between the two groups with respect to education level and marital status, with all other demographic data being comparable. A larger percentage of the intervention patients indicated to have a college or graduate degree (100\% vs. $68.4 \%)$ and identified themselves as married or partnered ( $84.2 \%$ vs. $47.4 \%$ ) compared to the usual care patients. Caregivers were comparable with regards to all demographic variables collected.

The majority of surgical procedures were minimally invasive (73.7\%). Mean length of stay was similar between groups ( 3.8 days for usual care, 3.2 for intervention). The majority of 
patients were treated for primary lung cancer $(78.9 \%)$. Usual care patients were more likely to undergo lobectomy ( $84 \%$ vs. $47 \%, \mathrm{p}<.005)$. There were no other significant group differences in patients' clinical and surgical characteristics (Table 3).

\section{Participant Outcomes}

For patients who received the MSM intervention, scores for total QOL improved, although the improvement was not statistically significant (Table 4). Emotional QOL was significantly improved post-intervention for the intervention group (19.9 vs. $15.9, \mathrm{p}=0.001$ ). This improvement is clinically meaningful, as a 3-point difference is considered a minimally important difference (MID). ${ }^{33}$ There were no significant improvements in total QOL and subscale scores for the usual care group. Pulmonary symptoms in the usual care group was significantly worse post-intervention $(\mathrm{p}=0.01)$. Surgery-related knowledge was significantly improved for patients in the intervention $(\mathrm{p}=0.003)$. There was a trend for significant improvement in self-efficacy $(\mathrm{p}=0.1)$ for the intervention group. Patient activation improved an average of 3.8 points post-intervention. Although this improvement was not statistically significant, this represents a clinically meaningful difference, as even a 3-point difference is considered a MID for confidence in managing health behaviors. ${ }^{34}$ Overall reliability was excellent for the PAM, with Cronbach's alpha measured at 0.92. Cronbach's alpha for the Self-Efficacy Scale was 0.89 . We did not observe statistically significant differences in preand post-intervention score changes for family caregivers overall. However, family caregiver surgery-related knowledge score was significantly improved post-intervention for both the usual care and intervention groups.

For healthcare resource use, 8 patients $(42.1 \%)$ in the intervention group and 7 patients $(36.8 \%)$ in the usual care group had an unscheduled outpatient encounter after surgery. For patients who received the MSM intervention, all unscheduled encounters were for triage phone calls. One patient in the usual care group had an urgent care/evaluation and treatment center visit (5.3\%). The majority of unscheduled encounters were for symptom management (36.8\%). Patients in the usual care group had more supportive care services referrals $(73.7 \%$ versus $42.1 \%$ ). These services included clinical nutrition, pain service, physical and pulmonary rehabilitation, clinical social work, and psychology/psychiatry. There were no statistically significant differences between-groups for healthcare resource use.

\section{Intervention Evaluations}

A total of 16 patients and 10 family caregivers completed the satisfaction tool to assess acceptability and usability of the intervention (26/30 intervention group participants, $86.7 \%$ ). Overall, participants have high-scaled evaluations for the intervention (Table 5). Mean scores for patient acceptability/usability rating for the intervention handbook and video was 3.6 to 3.7/4.0. Intervention evaluations from family caregivers were similar to patients (3.1 to 3.6/4.0), although there was input that more content on supporting caregivers' emotional well-being and self-management should be included. Eighty-seven percent of patients felt that the amount of intervention content was just right, and all patients felt comfortable with the timing of the intervention. Thirty percent of family caregivers felt that there was too little intervention content. 


\section{Discussion}

The MSM intervention, Preparing for Lung Surgery, was designed to prepare patients and family caregivers for lung surgery and improve outcomes using a systematically-developed self-management approach. ${ }^{17}$ While it may seem intuitive that there should be evidencebased, standardized preparatory interventions in cancer surgery, very few exist in the current literature. This may be secondary to several reasons: 1) time constraints for surgical teams, 2) lack of reimbursement for cancer educational and preparatory services, and 3) lack of research efforts to design evidence-based interventions. As hospital stays have shortened, so has the time available for preparing patients and families for lung surgery and postoperative recovery. Current perioperative and postoperative care results in rapid transitions to recovery at home and lack of formal assessment mechanisms between hospital discharge to first postoperative clinic visit. The goal of the MSM intervention was to improve surgical care by supporting the physical and psychosocial readiness and preparedness of patients and families for early discharge and recovery at home. Our intervention is novel because it includes family caregivers. This design recognizes that patients and families are a unit, and interventions in the peri-operative setting should address the needs of both.

One of the major goals of the MSM intervention was to empower and enhance patients' and family caregivers' control of their own care. Empowerment, or "activation," is a key construct of self-management models of care, and the MSM intervention was developed using this framework. While our pilot study was designed to assess feasibility and acceptability of the intervention, we were able to observe trends for improvement in outcomes such as emotional QOL, self-efficacy, activation, and knowledge. The improvements in these outcomes suggest that patients and family caregivers gained confidence, skills, and knowledge in managing their health. Improvements in self-efficacy, knowledge, and activation are likely necessary for improvements in other key outcomes such as QOL and healthcare resource use. The current evidence on self-management models of care supports this concept. Evidence for these mechanisms are found in studies with chronic illnesses such as COPD ${ }^{35}$, diabetes ${ }^{36}$, and more recently, cancer survivorship. ${ }^{37}$ Importantly, we found that participants generally derived benefits from the MSM intervention as indicated by their scores on acceptability and usability. Overall, the intervention scored highly for content, usefulness, and timing of the intervention.

Several important lessons could be noted from our pilot experience. First, although family caregivers were overall satisfied with the intervention, several felt that overall there were too little content to support caregiving needs. This deficiency may have resulted in our findings where we did not observe significant improvements in family caregiver outcomes. In response to the input, we plan on expanding family caregiver content across all components of the intervention to more comprehensively address self-care and include content to promote problem-solving skills. The problem-solving approach will include three key components: 1) identification of perceived barriers to caregiving, 2) prior plans or strategies to overcome these barriers, and 3) identify new strategies that are adoptable to foster positive caregiving experience and promote family caregiver self-care. The problem-solving coaching will be expanded throughout the intervention sessions to support caregiver skillbuilding. Second, we encountered challenges to implementing the different sessions of the 
MSM intervention, particularly before surgery. All of our participants were enrolled approximately 3-7 days prior to surgery. We were able to successfully accomplish this through close collaborations with our thoracic surgery team. We utilized routine preoperative outpatient clinic visits to deliver the intervention, thus eliminating the burden of an additional visit for patients and families.

This study has several limitations that must be noted. First, this was a pilot feasibility study, and our participant numbers were small. Therefore, our analysis was limited to feasibility and acceptability, and pre-and post-intervention comparisons were exploratory in nature. The study was not powered to determine differences between groups for key outcomes, and perhaps would have shown greater statistical significance with larger numbers of participants. Second, our results are likely biased because of challenges with including a more diverse population. Nevertheless, we were able to enroll approximately $38 \%$ ethnic minorities overall. Third, as patient and family caregiver QOL likely improves with time after surgery ${ }^{2}$, it is not entirely possible with our current findings to claim definitively that the observed improvements are due to the intervention alone. Based on our experience with this pilot study, we submitted an NCI R01 application to test the effects of the intervention in a large, 5-year, randomized trial. The RCT will focus on lung cancer patients with family caregivers only, and will explore mediators and moderators of dyadic outcomes and reciprocal relationships. In addition, we will determine the effects of the intervention on patient healthcare resource use and surgical outcomes (discharge disposition, readmissions).

\section{Conclusion}

The MSM intervention is a novel, multi-component (traditional information and technical skills plus self-management education), multimedia (audio/visual plus print) intervention focused on readiness and preparedness for lung surgery and postoperative recovery. We now have evidence to report that the MSM intervention is feasible and acceptable for patients and family caregivers. We also have exploratory evidence that demonstrates the potential impact of the intervention on emotional QOL, self-efficacy, activation, and knowledge. This will set the stage for a large randomized trial to determine the efficacy of the intervention on patient outcomes, family caregiver outcomes, and healthcare resource use.

\section{Acknowledgments}

The research reported in this paper included work performed in the Survey Research Core and Biostatistics Core that are supported by the National Cancer Institute of the National Institutes of Health under award number P30CA33572. The content is solely the responsibility of the authors and do not necessarily represent the official views of the NCI or NIH.

\section{References}

1. Miller KD, Siegel RL, Lin CC, Mariotto AB, Kramer JL, Rowland JH, Stein KD, Alteri R, Jemal A. Cancer treatment and survivorship statistics, 2016. CA Cancer J Clin. 2016; 66:271-289. [PubMed: 27253694]

2. Poghosyan H, Sheldon LK, Leveille SG, Cooley ME. Health-related quality of life after surgical treatment in patients with non-small cell lung cancer: A systematic review. Lung Cancer. 2013; 81:11-26. [PubMed: 23562675] 
3. Rauma V, Sintonen H, Rasanen JV, Salo JA, Ilonen IK. Long-term lung cancer survivors have permanently decreased quality of life after surgery. Clin Lung Cancer. 2015; 16:40-45. [PubMed: 25450878]

4. Wyld L, Audisio RA, Poston GJ. The evolution of cancer surgery and future perspectives. Nat Rev Clin Oncol. 2015; 12:115-124. [PubMed: 25384943]

5. Pereira L, Figueiredo-Braga M, Carvalho IP. Preoperative anxiety in ambulatory surgery: The impact of an empathic patient-centered approach on psychological and clinical outcomes. Patient Educ Couns. 2016; 99:733-738. [PubMed: 26654958]

6. Ronco M, Iona L, Fabbro C, Bulfone G, Palese A. Patient education outcomes in surgery: A systematic review from 2004 to 2010. International journal of evidence-based healthcare. 2012; 10:309-323. [PubMed: 23173656]

7. Johansson K, Nuutila L, Virtanen H, Katajisto J, Salantera S. Preoperative education for orthopaedic patients: Systematic review. J Adv Nurs. 2005; 50:212-223. [PubMed: 15788086]

8. Fujinami R, Otis-Green S, Klein L, Sidhu R, Ferrell B. Quality of life of family caregivers and challenges faced in caring for patients with lung cancer. Clinical Journal of Oncology Nursing. 2012; 16:E210-E220. [PubMed: 23178364]

9. Fujinami R, Sun V, Zachariah F, Uman G, Grant M, Ferrell B. Family caregivers' distress levels related to quality of life, burden, and preparedness. Psycho-oncology. 2015; 24:54-62. [PubMed: 24789500]

10. Grant M, Sun V, Fujinami R, Sidhu R, Otis-Green S, Juarez G, Klein L, Ferrell B. Family caregiver burden, skills preparedness, and quality of life in non-small cell lung cancer. Oncology nursing forum. 2013; 40:337-346. [PubMed: 23803267]

11. Kim JY, Sun V, Raz DJ, Williams AC, Fujinami R, Reckamp K, Koczywas M, Cristea M, Hurria A, Ferrell B. The impact of lung cancer surgery on quality of life trajectories in patients and family caregivers. Lung Cancer. 2016; 101:35-39. [PubMed: 27794406]

12. Jerofke TA, Weiss M, Yakusheva O. Patient perceptions of patient-empowering nurse behaviors, patient activation and functional health status in postsurgical patients with life-threatening longterm illness. Journal of Advanced Nursing. 2013; 70:1310-1322.

13. Taylor C, Richardson A, Cowley S. Restoring embodied control following surgical treatment for colorectal cancer: A longitudinal qualitative study. International journal of nursing studies. 2010; 47:946-956. [PubMed: 20096839]

14. McMurray A, Johnson P, Wallis M, Patterson E, Griffiths S. General surgical patients' perspectives of the adequacy and appropriateness of discharge planning to facilitate health decision-making at home. Journal of clinical nursing. 2007; 16:1602-1609. [PubMed: 17727581]

15. Lapum J, Angus JE, Peter E, Watt-Watson J. Patients' discharge experiences: Returning home after open-heart surgery. Heart \& lung : the journal of critical care. 2011; 40:226-235. [PubMed: 20561868]

16. McCorkle R, Ercolano E, Lazenby M, Schulman-Green D, Schilling LS, Lorig K, Wagner EH. Self-management: Enabling and empowering patients living with cancer as a chronic illness. CA Cancer J Clin. 2011; 61:50-62. [PubMed: 21205833]

17. Sun V, Kim JY, Raz DJ, Chang W, Erhunmwunsee L, Uranga C, Ireland AM, Reckamp K, Tiep B, Hayter J, Lew M, Ferrell B, McCorkle R. Preparing cancer patients and family caregivers for lung surgery: Development of a multimedia self-management intervention. Journal of cancer education : the official journal of the American Association for Cancer Education. 2016

18. Gysels M, Higginson IJ. Interactive technologies and videotapes for patient education in cancer care: Systematic review and meta-analysis of randomised trials. Supportive care in cancer : official journal of the Multinational Association of Supportive Care in Cancer. 2007; 15:7-20. [PubMed: 17024500]

19. Coleman K, Austin BT, Brach C, Wagner EH. Evidence on the chronic care model in the new millennium. Health affairs. 2009; 28:75-85. [PubMed: 19124857]

20. Wagner EH, Bennett SM, Austin BT, Greene SM, Schaefer JK, Vonkorff M. Finding common ground: Patient-centeredness and evidence-based chronic illness care. Journal of alternative and complementary medicine. 2005; 11(Suppl 1):S7-S15. [PubMed: 16332190] 
21. Wagner EH, Austin BT, Davis C, Hindmarsh M, Schaefer J, Bonomi A. Improving chronic illness care: Translating evidence into action. Health affairs. 2001; 20:64-78. [PubMed: 11816692]

22. Mosen DM, Schmittdiel J, Hibbard J, Sobel D, Remmers C, Bellows J. Is patient activation associated with outcomes of care for adults with chronic conditions? The Journal of ambulatory care management. 2007; 30:21-29. [PubMed: 17170635]

23. Hibbard JH, Stockard J, Mahoney ER, Tusler M. Development of the patient activation measure (pam): Conceptualizing and measuring activation in patients and consumers. Health services research. 2004; 39:1005-1026. [PubMed: 15230939]

24. Lorig KR, Holman H. Self-management education: History, definition, outcomes, and mechanisms. Annals of behavioral medicine : a publication of the Society of Behavioral Medicine. 2003; 26:17. [PubMed: 12867348]

25. Grey M, Schulman-Green D, Knafl K, Reynolds NR. A revised self- and family management framework. Nursing outlook. 2015; 63:162-170. [PubMed: 25771190]

26. Cella DF, Tulsky DS, Gray G, Sarafian B, Linn E, Bonomi A, Silberman M, Yellen SB, Winicour $\mathrm{P}$, Brannon J. The functional assessment of cancer therapy scale: Development and validation of the general measure. Journal of Clinical Oncology. 1993; 11:570-579. [PubMed: 8445433]

27. Cleeland CS, Mendoza TR, Wang XS, Chou C, Harle MT, Morrissey M, Engstrom MC. Assessing symptom distress in cancer patients: The m.D. Anderson symptom inventory. Cancer. 2000; 89:1634-1646. [PubMed: 11013380]

28. Magasi S, Mallick R, Kaiser K, Patel JD, Lad T, Johnson ML, Kaplan EH, Cella D. Importance and relevance of pulmonary symptoms among patients receiving second- and third-line treatment for advanced non-small-cell lung cancer: Support for the content validity of the 4-item pulmonary symptom index. Clinical lung Cancer. 2013; 14:245-253. [PubMed: 23036663]

29. Lorig K, Chastain RL, Ung E, Shoor S, Holman HR. Development and evaluation of a scale to measure perceived self-efficacy in people with arthritis. Arthritis and rheumatism. 1989; 32:37-44. [PubMed: 2912463]

30. Ferrell BR, Grant M, Borneman T, Juarez G, ter Veer A. Family caregiving in cancer pain management. Journal of palliative medicine. 1999; 2:185-195. [PubMed: 15859815]

31. Ferrell BR, Grant M, Chan J, Ahn C, Ferrell BA. The impact of cancer pain education on family caregivers of elderly patients. Oncology nursing forum. 1995; 22:1211-1218. [PubMed: 8532545]

32. Montgomery RV, Stull DE, Borgatta EF. Measurement and the analysis of burden. Research on aging. 1985; 7:137-152. [PubMed: 4059631]

33. Cella DF, Tulsky DS, Gray G, Sarafian B, Linn E, Bonomi A, Silberman M, Yellen SB, Winicour $\mathrm{P}$, Brannon J, et al. The functional assessment of cancer therapy scale: Development and validation of the general measure. Journal of clinical oncology : official journal of the American Society of Clinical Oncology. 1993; 11:570-579. [PubMed: 8445433]

34. Fowles JB, Terry P, Xi M, Hibbard J, Bloom CT, Harvey L. Measuring self-management of patients' and employees' health: Further validation of the patient activation measure (pam) based on its relation to employee characteristics. Patient Educ Couns. 2009; 77:116-122. [PubMed: 19356881]

35. Benzo R, Vickers K, Novotny PJ, Tucker S, Hoult J, Neuenfeldt P, Connett J, Lorig K, McEvoy C. Health coaching and copd re-hospitalization: A randomized study. American journal of respiratory and critical care medicine. 2016

36. Lorig K, Ritter PL, Turner RM, English K, Laurent DD, Greenberg J. Benefits of diabetes selfmanagement for health plan members: A 6-month translation study. Journal of medical Internet research. 2016; 18:e164. [PubMed: 27342265]

37. Salvatore AL, Ahn S, Jiang L, Lorig K, Ory MG. National study of chronic disease selfmanagement: 6-month and 12-month findings among cancer survivors and non-cancer survivors. Psychooncology. 2015; 24:1714-1722. [PubMed: 25871889] 


\section{Clinical Practice Points}

Surgery is a key component in the treatment for most patients with non-metastatic lung cancer, but by its very nature, lung resection can significantly worsen QOL. Patients experience pain, fatigue, loss of respiratory capacity, and decreased physical function after lung cancer surgery. Family caregivers experience significant psychological distress and decreased QOL related to their caregiving role. The current literature offers few evidence-based, patient-centered models of care in cancer surgery. Taking into account the critical importance of this unmet need, a multimedia self-management intervention to prepare patients and family caregivers for lung surgery and post-operative recovery was developed and pilot-tested. We found the intervention to be feasible and acceptable for patients and family caregivers, with potential positive impact on emotional QOL, selfefficacy, activation, and knowledge. This intervention has the potential to transform the quality of surgical care for cancer patients and their families. 


\section{Table 1}

\section{MSM Intervention Content}

\begin{tabular}{|c|l|}
\hline \multicolumn{1}{|c|}{ Session 1 } & Understanding Your Operation \\
What to Expect Before Surgery & Staying Smoke Free \\
What to Expect on the Day of Surgery & Breathing Exercises (Incentive Spirometry) \\
What to Expect After Surgery - & Staying Active Before Surgery \\
Recovery in the Hospital & Coping with Anxiety \\
& Pre-operative clinic \\
& Day of Surgery Admissions \\
\cline { 2 - 3 } & Pain Assessment and Management \\
& Chest Tube \\
& Breathing Exercises (Incentive Spirometry) \\
& Early Ambulation \\
\hline \multirow{2}{*}{ Session 2 } & Pain Assessment and Management \\
& Cough and Breathing \\
& Nutrition \\
& Activities, Intimacy, Fatigue, Return to Work \\
& Sleep \\
& When to Call Your Doctor \\
\hline Session 3 \& 4: & Pain Assessment and Management \\
& Pulmonary Symptoms \\
& Activities and Nutrition \\
\hline
\end{tabular}


Sun et al.

Page 14

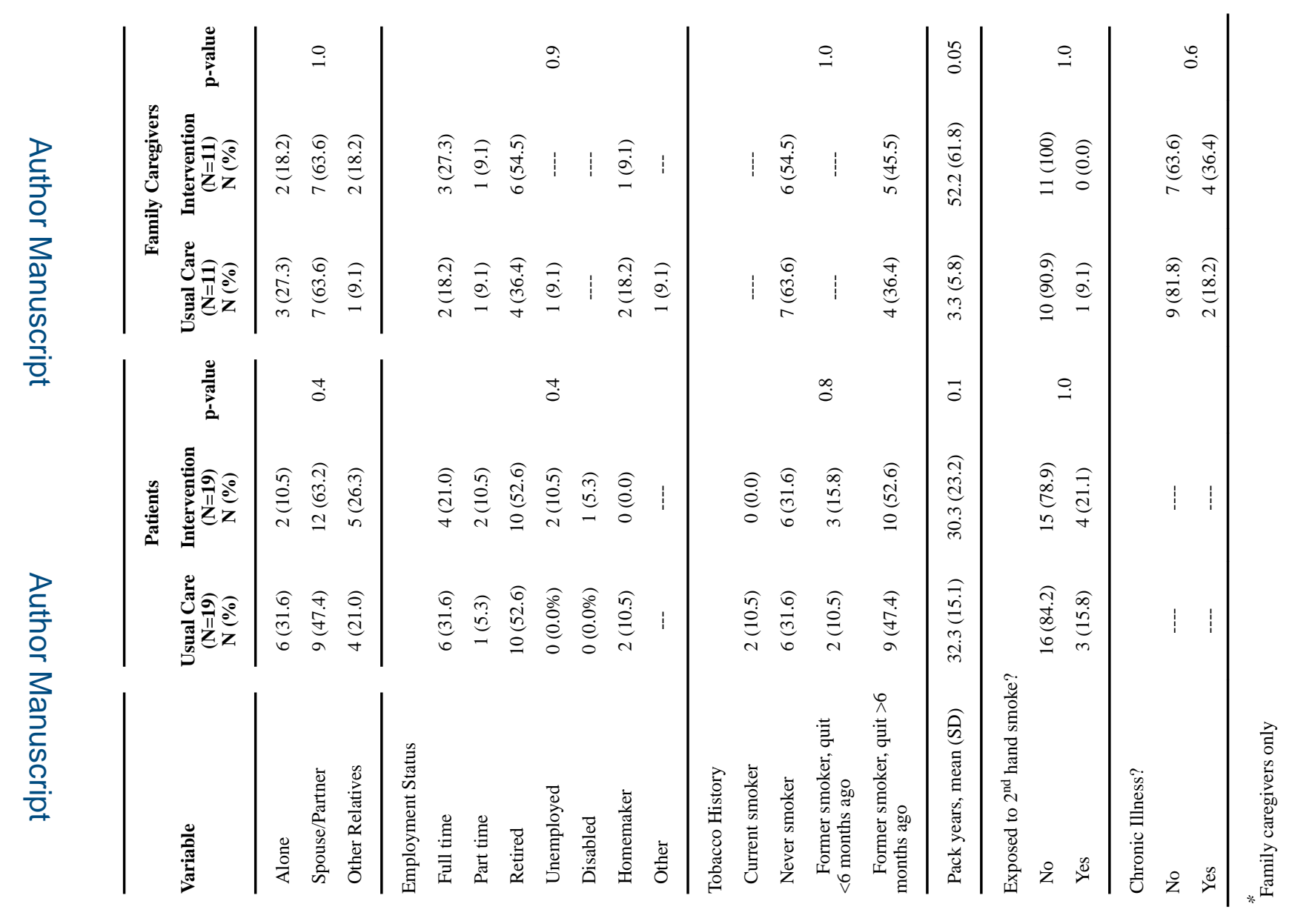

로을

로을

Clin Lung Cancer. Author manuscript; available in PMC 2018 May 01. 
Table 3

Clinical and Surgical Characteristics

\begin{tabular}{|c|c|c|c|}
\hline & $\begin{array}{c}\text { Control } \\
(\mathbf{n}=19)\end{array}$ & $\begin{array}{c}\text { Intervention } \\
(\mathrm{n}=19)\end{array}$ & p-value \\
\hline Pre-op weight $(\mathrm{kg})$, mean $(\mathrm{SD})$ & $79.4(21.4)$ & $79.8(19.0)$ & 1.0 \\
\hline \multicolumn{4}{|l|}{ Co-morbidities/Pre-op Risk Factors, $N(\%)$ yes } \\
\hline Hypertension & $11(57.9)$ & $6(31.6)$ & 0.1 \\
\hline Coronary Artery Disease & $3(15.8)$ & $1(5.3)$ & 0.6 \\
\hline Prior Cardiothoracic Surgery & $2(10.5)$ & $0(0.0)$ & 0.5 \\
\hline Neoadjuvant chemotherapy & $2(10.5)$ & $4(21.1)$ & 0.7 \\
\hline Treated $<=6 \mathrm{mo}$ before surgery & $2(10.5)$ & $4(21.1)$ & 0.7 \\
\hline Preoperative radiation & $1(5.3)$ & $0(0.0)$ & NA \\
\hline Diabetes & $5(26.3)$ & $3(15.8)$ & 0.7 \\
\hline Chronic Obstructive Pulmonary Disease & $3(15.8)$ & $3(15.8)$ & NA \\
\hline \multicolumn{4}{|l|}{ Pulmonary Function } \\
\hline FEV1 \% predicted, mean $(\mathrm{SD})$ & $93.3(22.7)$ & $92.5(21.6)$ & 0.9 \\
\hline DLCO \% predicted, mean (SD) & $83.3(12.7)$ & $89.3(17.6)$ & 0.3 \\
\hline \multicolumn{4}{|l|}{ Pre-op Zubrod Score, N (\%) } \\
\hline 0 - Normal activity, no symptoms & $12(63.2)$ & $15(78.9)$ & \multirow{4}{*}{0.4} \\
\hline 1 - Symptoms, fully ambulatory & $5(26.3)$ & $1(5.3)$ & \\
\hline 2 - Symptoms in bed, $<=50 \%$ of the time & $1(5.3)$ & $2(10.5)$ & \\
\hline 3- Symptoms in bed, 50 to $<100 \%$ of the time & $1(5.3)$ & $1(5.3)$ & \\
\hline \multicolumn{4}{|l|}{ Surgical Approach } \\
\hline Minimally Invasive & $16(84.2)$ & $12(63.1)$ & \multirow{2}{*}{0.1} \\
\hline Open & $3(15.8)$ & $7(36.8)$ & \\
\hline \multicolumn{4}{|l|}{ Category of lung primary, $\mathrm{N}(\%)$} \\
\hline Lung cancer, NSCLC & $17(89.5)$ & $13(68.4)$ & \multirow{2}{*}{0.2} \\
\hline Lung tumor, metastatic & $2(10.5)$ & $6(31.6)$ & \\
\hline \multicolumn{4}{|l|}{ Primary Procedure, N (\%) } \\
\hline Wedge Resection & $2(10.5)$ & $8(42.1)$ & \multirow{4}{*}{0.005} \\
\hline Lobectomy & $16(84.2)$ & $9(47.4)$ & \\
\hline Bilobectomy & $0(0.0)$ & $1(5.3)$ & \\
\hline Pneumonectomy & $1(5.3)$ & $1(5.3)$ & \\
\hline Length of Stay (days), mean (SD) & $3.8(2.2)$ & $3.2(2.0)$ & 0.3 \\
\hline \multicolumn{4}{|l|}{ Discharge Disposition, N (\%) } \\
\hline Home & $18(94.7)$ & $18(94.7)$ & \multirow{2}{*}{1.0} \\
\hline Extended care & $1(5.3)$ & $1(5.3)$ & \\
\hline \multicolumn{4}{|l|}{30 day Readmission, $\mathbf{N}(\%)$} \\
\hline Yes & $1(5.3)$ & $3(15.8)$ & 0.3 \\
\hline Return to OR, N (\%) & $4(21.2)$ & $0(0.0)$ & 0.04 \\
\hline
\end{tabular}




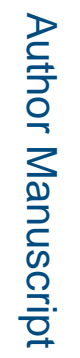

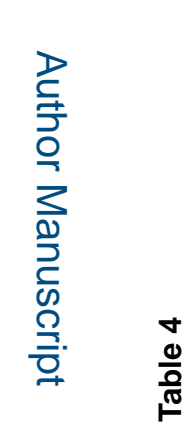

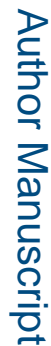

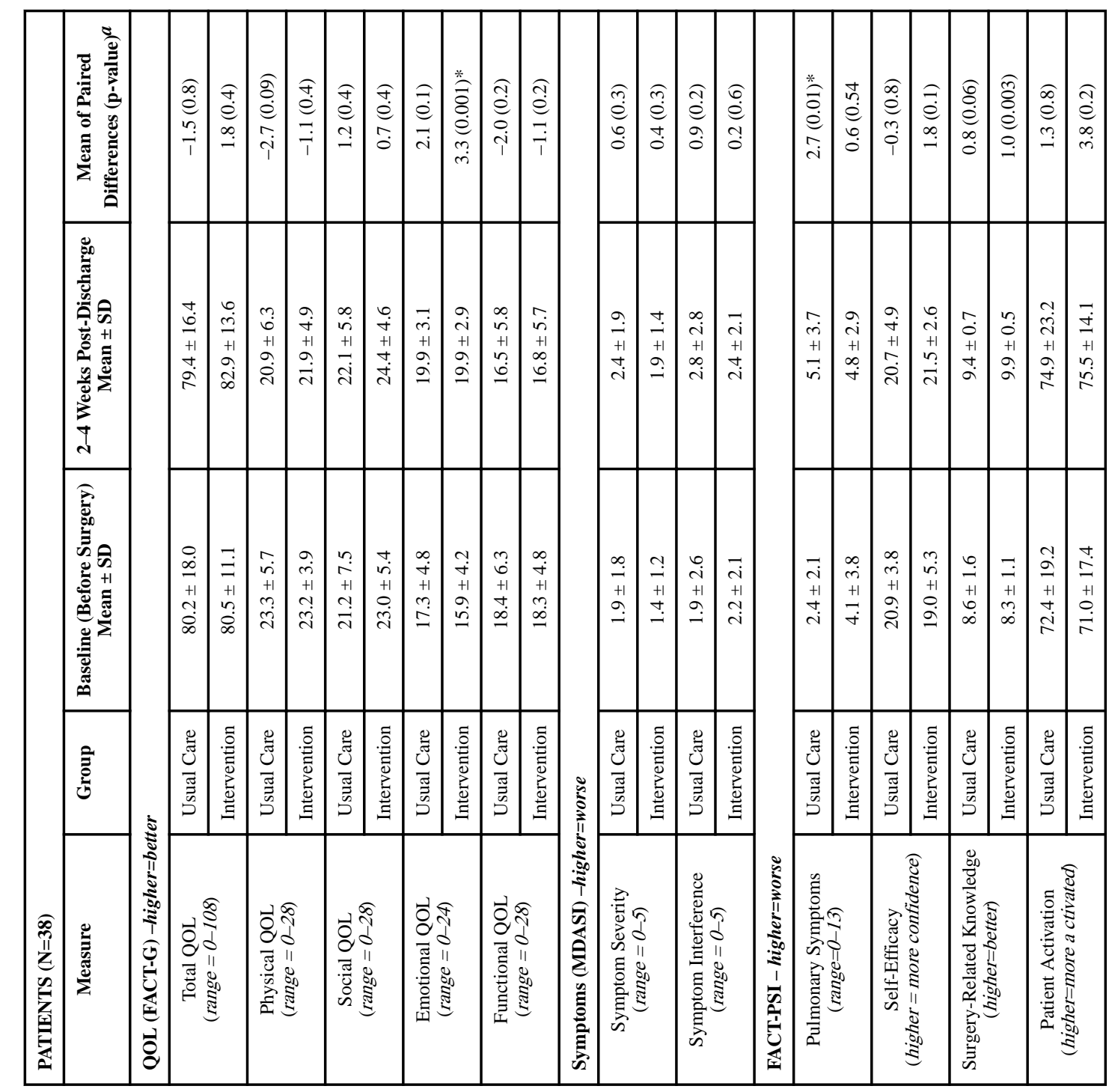

Clin Lung Cancer. Author manuscript; available in PMC 2018 May 01. 


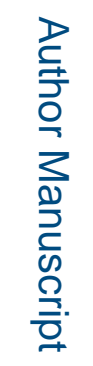

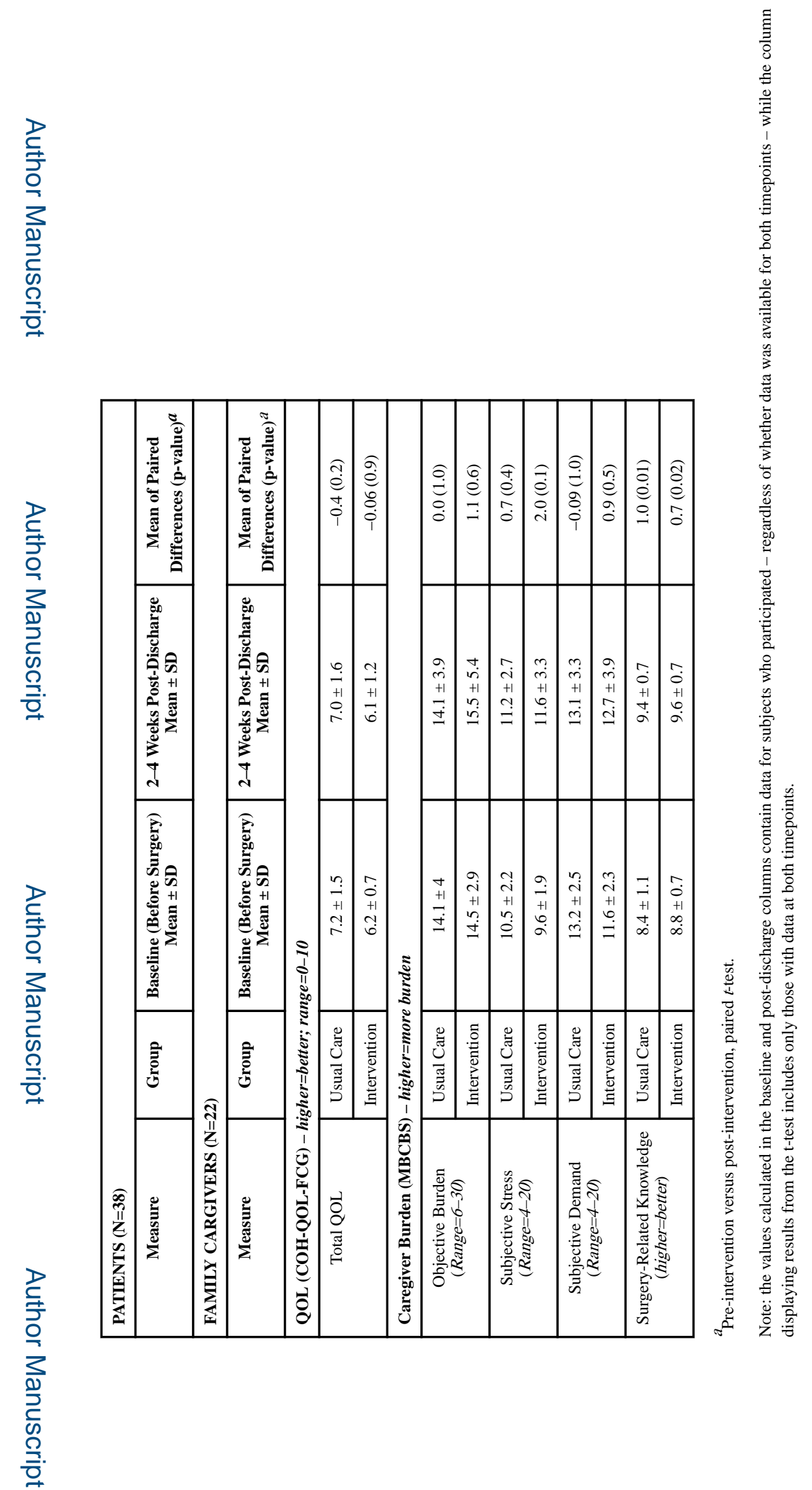

Clin Lung Cancer. Author manuscript; available in PMC 2018 May 01. 
Table 5

Intervention Evaluations

\begin{tabular}{|c|c|c|c|c|}
\hline \multirow[b]{2}{*}{ Item } & \multicolumn{2}{|c|}{ Patients $(\mathrm{N}=16)$} & \multicolumn{2}{|c|}{ Family Caregivers $(\mathrm{N}=10)$} \\
\hline & $\overline{\mathbf{x}}$ & SD & $\overline{\mathbf{x}}$ & SD \\
\hline \multicolumn{5}{|c|}{$\begin{array}{l}\text { Acceptability/Usability of Intervention } \\
\text { Higher score = higher satisfaction; range= }=-4\end{array}$} \\
\hline \multicolumn{5}{|l|}{ Overall Acceptability Rating } \\
\hline Handbook & 3.6 & 0.6 & 3.3 & 0.7 \\
\hline Video & 3.6 & 0.6 & 3.1 & 0.7 \\
\hline \multicolumn{5}{|l|}{ Overall Usability Rating } \\
\hline Handbook & 3.7 & 0.6 & 3.6 & 0.5 \\
\hline Video & 3.7 & 0.6 & 3.2 & 0.8 \\
\hline \multicolumn{5}{|l|}{ Usability of Handbook } \\
\hline Instructions & 3.4 & 0.6 & 3.2 & 0.8 \\
\hline Preparing family for surgery & 3.4 & 0.9 & 3.3 & 0.5 \\
\hline What to expect before surgery & 3.4 & 0.9 & 3.4 & 0.7 \\
\hline What to expect day of surgery & 3.3 & 1.0 & 3.5 & 0.7 \\
\hline Pain assessment and management & 3.4 & 0.8 & 3.4 & 0.5 \\
\hline Breathing exercises & 3.7 & 0.5 & 3.5 & 0.5 \\
\hline Walking/ambulation & 3.6 & 0.6 & 3.4 & 0.7 \\
\hline What to expect after discharge & 3.5 & 0.6 & 3.5 & 0.5 \\
\hline When to contact MD & 3.3 & 0.7 & 3.4 & 0.7 \\
\hline Additional resources & 2.8 & 1.2 & 3.0 & 1.1 \\
\hline \multicolumn{5}{|l|}{ Usability of Video } \\
\hline Instructions & 3.4 & 0.9 & 3.1 & 0.9 \\
\hline Preparing family for surgery & 3.3 & 0.9 & 3.3 & 0.8 \\
\hline What to expect before surgery & 3.4 & 0.9 & 3.1 & 0.9 \\
\hline What to expect day of surgery & 3.4 & 0.9 & 3.2 & 0.8 \\
\hline What to expect after surgery & 3.6 & 0.6 & 3.2 & 0.8 \\
\hline What to expect after discharge & 3.5 & 0.6 & 3.4 & 0.8 \\
\hline \multicolumn{5}{|c|}{ Amount of content in video and handbook - N (\%) } \\
\hline Too little & \multicolumn{2}{|c|}{$1(6.3)$} & \multicolumn{2}{|c|}{$3(30.0)$} \\
\hline Just right & \multicolumn{2}{|c|}{$14(87.5)$} & \multicolumn{2}{|c|}{$7(70.0)$} \\
\hline Too much & \multicolumn{2}{|c|}{$1(6.3)$} & \multicolumn{2}{|c|}{----} \\
\hline \multicolumn{5}{|l|}{ Timing of intervention $-\mathrm{N}(\%)$} \\
\hline Just right & \multicolumn{2}{|c|}{$16(100)$} & \multicolumn{2}{|c|}{$10(100.0)$} \\
\hline
\end{tabular}

